\title{
Rapid Laser Direct Writing of Plasmonic Components
}

\author{
G. Amoako ${ }^{1,2}$, W. Zhang ${ }^{1}$, M. Zhou ${ }^{1}$, S. S. Sackey ${ }^{2}$ \& P. Mensah-Amoah ${ }^{2}$ \\ 1. Center for Photon Manufacturing Science and Technology, Jiangsu University, Zhenjiang, Jiangsu, P. R. China \\ 2. Department of Physics, University of Cape Coast, Cape Coast, Ghana \\ Correspondence: G. Amoako, Center for Photon Manufacturing Science and Technology, Jiangsu University, \\ Zhenjiang, Jiangsu, 212013, P. R. China. Tel: 233-505-300-062. E-mail: gamoako@ucc.edu.gh
}

Received: October 21, 2017

Accepted: October 29, 2017

Online Published: November 7, 2017

doi:10.5539/apr.v9n6p19

URL: https://doi.org/10.5539/apr.v9n6p19

\begin{abstract}
A new device named technology-plasmonics has recently emerged and can be used to manipulate light at the nanoscale level. Here, we report the method of two-photon photopolymerization for rapid laser direct writing of plasmonic components. The characterization of these components is performed by a leakage radiation microscope, which has the same system construction as the two-photon photopolymerization micro-fabrication system except the laser pattern. The dielectric structures covered with gold proved to be very efficient for the excitation of surface plasmon polaritons in this system and can achieve different plasmon fields.
\end{abstract}

Keywords: plasmonic, surface plasmons, thin films, numerical aperture, waveguide

\section{Introduction}

The current trend towards miniaturization of optical components and devices require methods of observation with high spatial resolutions in the micron or sub-micron optical region. However, conventional optics is diffractionlimited to about half of the optical wavelength (Barnes et al., 2003). Surface plasmon polaritons (SPPs) excited on nanostructured metal-dielectric interfaces turn out to be the promising candidate to overcome the classical diffraction limit (Raether, 1988). SPPs are surface electromagnetic waves that propagate at a metal-dielectric interface and exhibit a strong coupling to the free electrons in the metal. Their electromagnetic field intensity is highest at the surface and decays exponentially away from the interface. If the interface is nanostructured, the spatial dimension of the new structures will determine the distribution of the SPPs field. SPP optical devices built at such an interface includes nanostructures like mirrors, beam splitters and quantum dots (Barnes et al., 2003; Drezet et al., 2005; Evlyukhin, Bozhevolnyi, Stepanov, \& Krenn, 2006; González et al., 2006).

Two-photon photopolymerization (TPP) of femtosecond laser is a rapid, cheap and flexible method to fabricate different microstructures such as three-dimensional woodpile photonic crystals with good quality (Sun, Matsuo, \& Misawa, 1999; Kaneko, Sun, Duan, \& Kawata, 2003; Qi, Lidorikis, \& Rakich, 2004; Deubel \& Freymann, 2004; Zhou, Kong, Yang, Yan, \& Cai, 2007) and plasmonic components (Reinhardt et al., 2006). In this paper we use TPP to fabricate different microstructures in the photosensitive resin of ORganically MOdified CERamic (ORMOCER) as the dielectric structure, which is covered with gold thin film to form the metal-dielectric interface for localization and guiding of SPPs.

We apply the far-field technique to SPP imaging by leakage radiation microscopy (LRM) (Hecht, Bielefeldt, Novotny, Inouye, \& Pohl, 1996; Bouhelier et al., 2001; Stepanov et al., 2005; Yang \& Zhou, 2013), which simplifies the experiment and accelerates the formation of the SPP intensity images. The leakage radiation relies on the light emitted by the SPPs and can radiate into leaky electromagnetic waves in the substrate based on the asymmetric environment (metal/dielectric). The dielectric structure can be designed to suppress the strong leakage radiation to reduce the SPP losses. Such a far-field optical method allows a direct quantitative imaging and analysis of SPP propagation on the metal-dielectric interfaces.

In this work we combine the TPP micro-fabrication system with the LRM system in the same experimental setup except the laser pattern. Based on such simplified setup, SPPs are excited and observed in different plasmonic components fabricated by TPP. The plasmonic component consisting of gold/ORMOCER/glass structure is shown in Figure 1. 


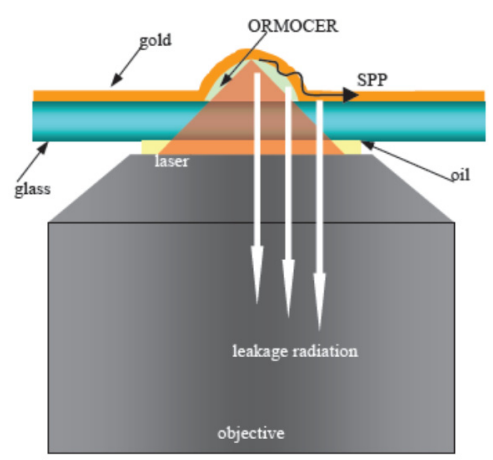

Figure 1. A sketch of the combination of the TPP micro-fabrication system and the LRM system

\section{Experiment}

For the fabrication of a structure as shown in Figure 1, the ORMOCER is first spun on the cover glass; the laser beam is focused on the ORMOCER to form the surface polymer structure by TPP and finally $50 \mathrm{~nm}$-thin gold films are deposited on the washed samples. The method of finite difference time domain (FDTD) is used to investigate the SPPs distribution in the formed structure (Müller, Ropers, \& Lienau, 2004; Chang \& Gray, 2005). In Figure 2, the left image is the gold/ORMOCER/glass structure with gold in the top layer, the ORMOCER is in the middle layer and the glass in the bottom layer. The system is irradiated with $800 \mathrm{~nm}$ femtosecond laser from the bottom. Simulation results, as shown in the right image of Figure 2, shows that SPPs are excited in the structure, with the strongest intensity in the interface between gold and ORMOCER.
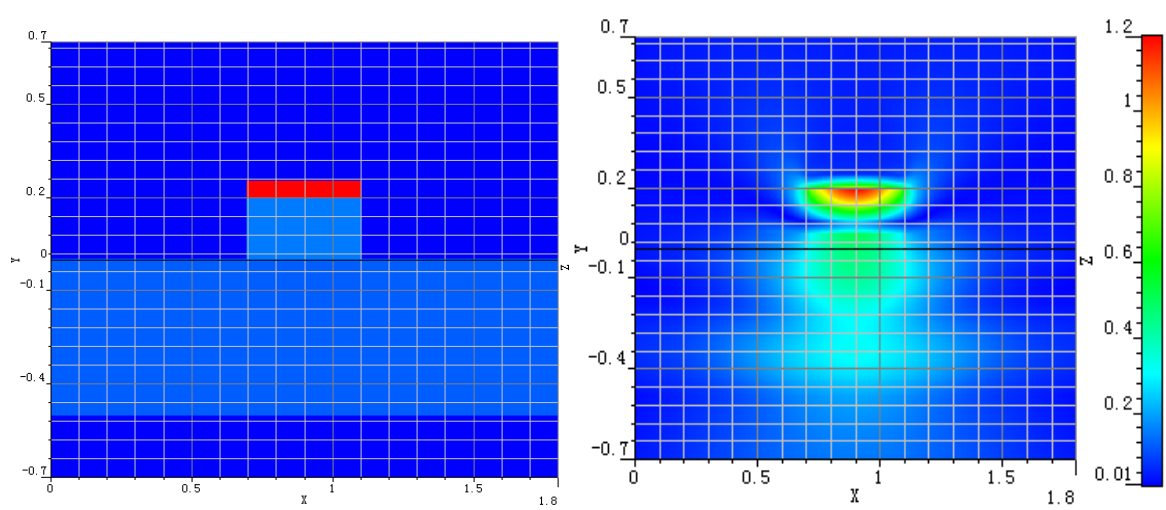

Figure2. A sketch of the $\mathrm{Au} /$ resin / glass structure (left) and a simulated distribution of the SPPs in the Au / resin / glass structure (right)

In the TPP micro-fabrication system, the femtosecond laser pulses (30 fs, $82 \mathrm{MHz}, 800 \mathrm{~nm}$ ) are tightly focused into the volume of the photosensitive resin ORMOCER with a high numerical aperture $(\mathrm{NA}=1.35)$ oil immersion objective, while in the LRM system, the femtosecond laser is a continuous laser with the wavelength of $800 \mathrm{~nm}$. The combined system acts like an inverted microscope with a laser source. A charge-coupled device (CCD) camera mounted behind a dichroic mirror in the system is used for online monitoring of the TPP process (Zhou, Kong, Yang, Yan, \& Cai, 2007) and excitation collection of the leakage radiation.

An atomic force microscope (AFM) is used for the visual inspection of the ORMOCER structure samples fabricated with TPP. Figure 3 and Figure 4 show AFM images of the micro-grating template and micro-crater template structures respectively. Such templates have good qualities and smooth surfaces and have the potential possibility for localization and guiding of SPPs. 


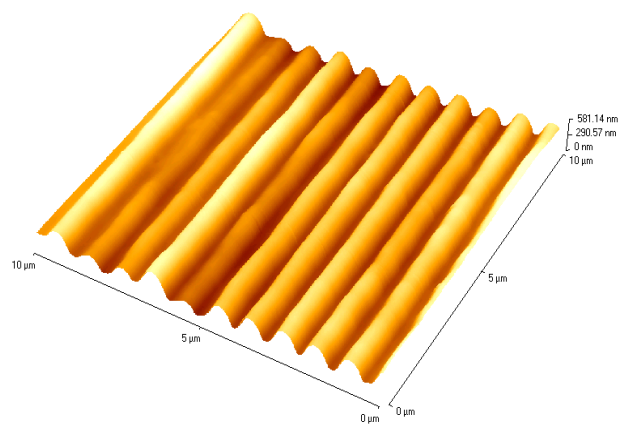

Figure 3.The micro-grating template

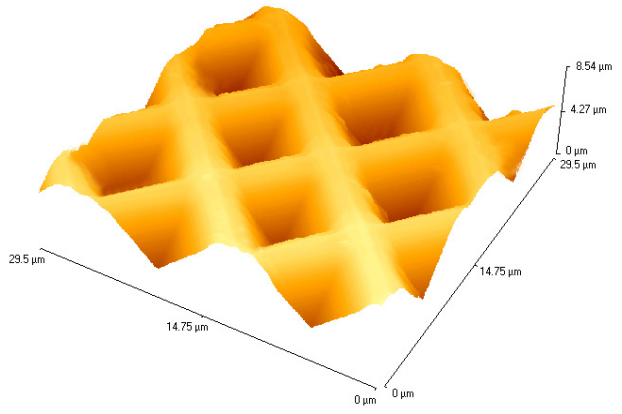

Figure 4.The micro-crater template

\section{SPP Excitation and Observation}

In the TPP process, the fabrication parameters are controlled using a numerical aperture 1.35 , laser power of 60 $\mathrm{mW}$ and the scanning speed of $80 \mu \mathrm{m} / \mathrm{s}$. For such arrangement the lateral rod diameters of the fabricated rods are typically in the range of $800 \mathrm{~nm}$ to $1000 \mathrm{~nm}$. The dielectric structures can be used to guide the SPPs. The structure under investigation is a straight waveguide with a rod diameter of about $3000 \mathrm{~nm}$. SPPs can be excited at a wavelength of $800 \mathrm{~nm}$ using a laser beam focused on one port (marked with a broken circle in Figure 5) of the waveguide while the propagation of the SPPs is observed on the other port, as shown in Figure 5. For such arrangement the non-radiative SPPs have not been blocked inside the waveguide structure with a relatively high intensity on the exit port due to the confinement and guiding of the ORMOCER waveguide. When the exciting laser beam is focused on the edge of the waveguide, the SPPs will spread across the waveguide and propagate forward as shown in Figure 6, where the SPPs are excited on the edge of the structure with a height of $4000 \mathrm{~nm}$ and a rod diameter of about $4000 \mathrm{~nm}$. They propagate over the smooth surface of the structure. This result clearly indicates that SPPs can propagate on smoothly curved surfaces with curvatures larger than the SPP wavelength. The distribution situation in Figure 5 and Figure 6 shows that excitation on different parts of the structure can produce different SPPs.

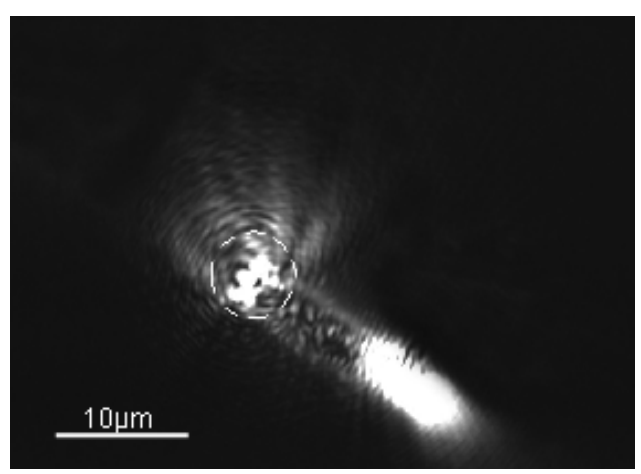

Figure 5. SPPs excited on one port of the waveguide

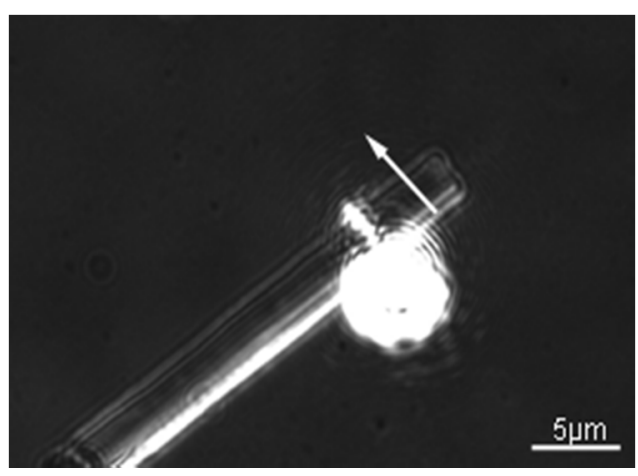

Figure 6. SPPs excited on the edge of the waveguide

We focus on different plasmonic components with right-angled and crossed-waveguide structures and discuss the excitation and transmission of the SPPs in such structures. Two right-angled waveguides are fabricated with side lengths of $10 \mu \mathrm{m}$ and $20 \mu \mathrm{m}$, as shown in Figure 7 and Figure 8 respectively. SPPs excited on one port of the rightangled waveguide propagate forward along the waveguide and weaken as the side lengths increase. There is a large loss of the SPPs in the corner of the right-angled waveguide. The loss increases further at the shorter side length. The regular distribution of SPPs can be observed on the other port of the right-angled waveguide. In Figure 7 and Figure 8, the correct images are the partially enlarged view of the ones in the frames of the left images, which show the SPPs distribution near the exit port in detail. In the side near the exit port of the waveguide, the stripes of SPPs are parallel to each other in the vertical direction to the transmission path. In the other part of the exit port, the SPPs are regular dot patterns due to electromagnetic interference. The stripes can be used to deduce the wavelength of the SPP, which is twice the length of the adjacent two-stripes. The transmission distance of the SPPs is at least $40 \mu \mathrm{m}$ long, as shown in Figure 8. If we continue to increase the length of the right-angled waveguide, the transmission distance of the SPPs may be longer. 


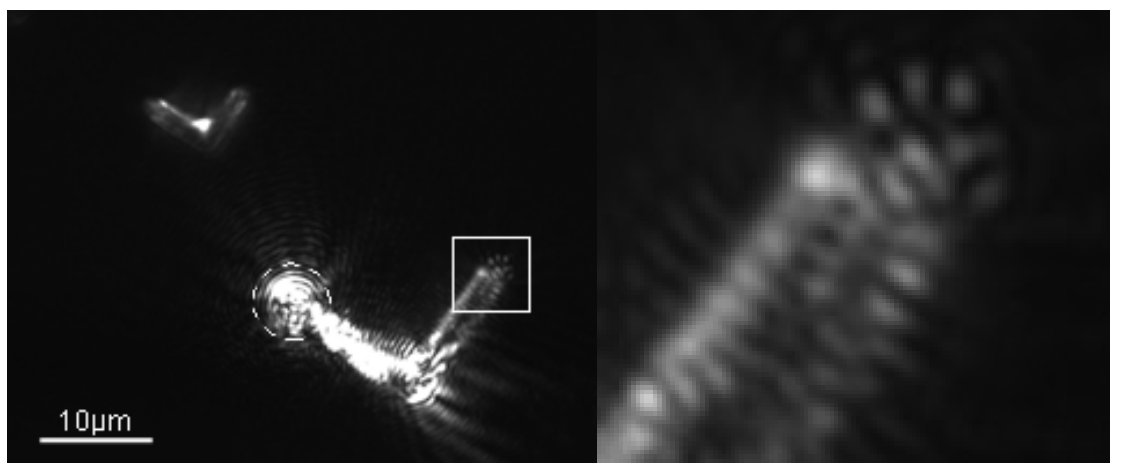

Figure 7. SPPs excited on one port of the right-angled structure with the side length of $10 \mu \mathrm{m}$ (left) and the partially enlarged view of the one in the frame of the left image (right)

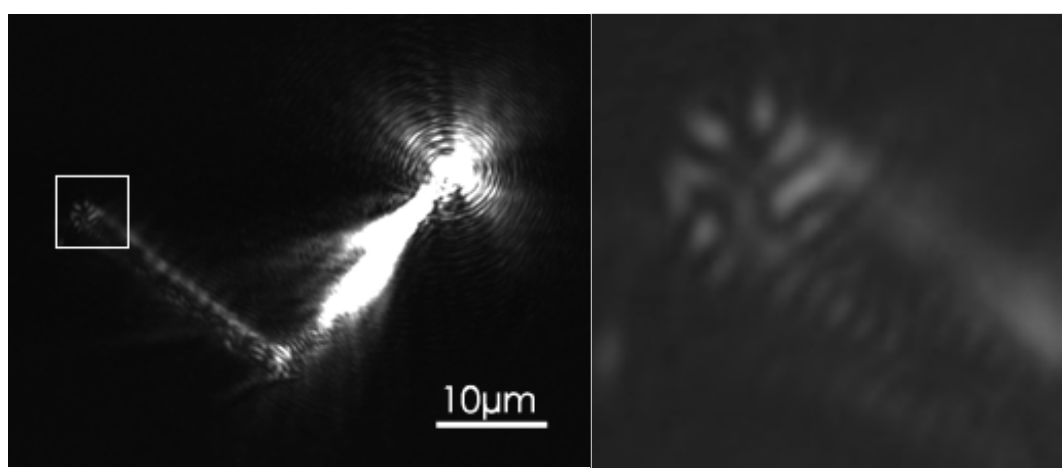

Figure 8. SPPs excited on one port of the right-angled structure with the side length of $20 \mu \mathrm{m}$ (left) and the partially enlarged view of the one in the frame of the left image (right)

The SPPs can also be excited on the corner of the right-angled waveguide, as shown in Figure 9 and Figure 10. The parallel stripes of the SPPs appear and propagate forward along the waveguide and weaken as the transmission distance increases. The $10 \mu \mathrm{m}$-long right-angled structure has a stronger SPPs distribution than the $20 \mu \mathrm{m}$-long one as shown in the right images in Figure 9 and Figure 10. The SPPs have the tendency to transmit forward after leaving the waveguide structure as shown in Figure 9 and Figure 10, while they do not show this trend to move forward as shown in Figure 7 and Figure 8. The results show that this structure can be used to control the localization and guiding of SPPs.

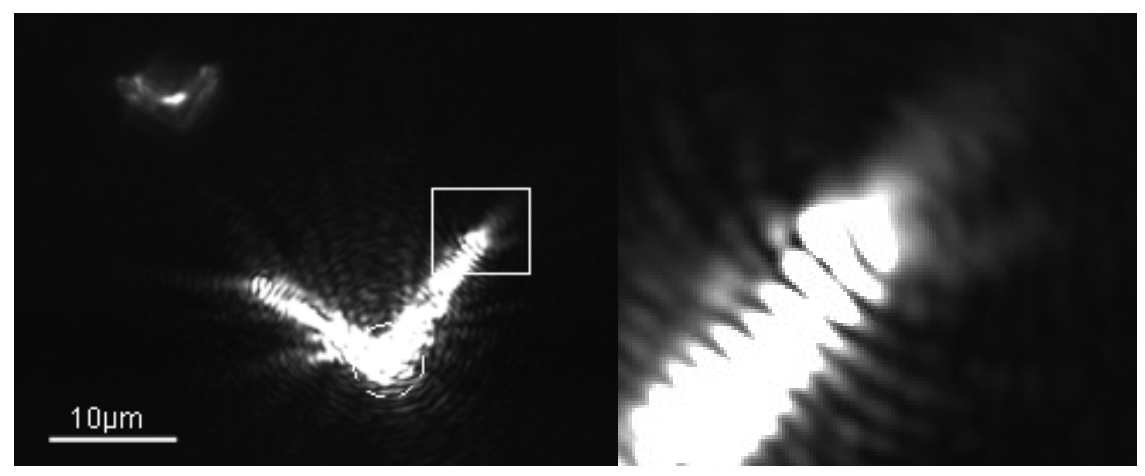

Figure 9. SPPs excited on the corner of the right-angled structure with the side length of $10 \mu \mathrm{m}$ (left) and the partially enlarged view of the one in the frame of the left image (right) 


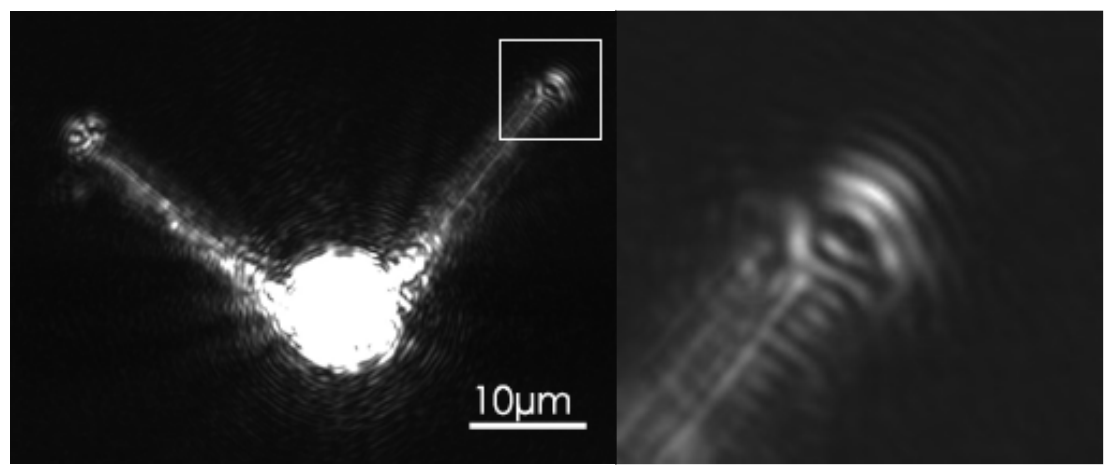

Figure 10. SPPs excited on the corner of the right-angled structure with the side length of $20 \mu \mathrm{m}$ (left) and the partially enlarged view of the one in the frame of the left image (right)

If the laser beam is focused on the crossed section of the crossed-waveguide, the excited SPPs will transmit forward along the four sides of the structure, as shown in the left image of Figure 11. When focused on one port of the crossed-waveguide, the SPPs will transmit forward along one side, past the cross, and spread to the other three sides, as shown in the right image of Figure 11. Excitation on different sections of the structure can produce different SPPs and different transmission trends. This allows us to design and fabricate different plasmonic components to control the SPP distribution in different parts of the components.

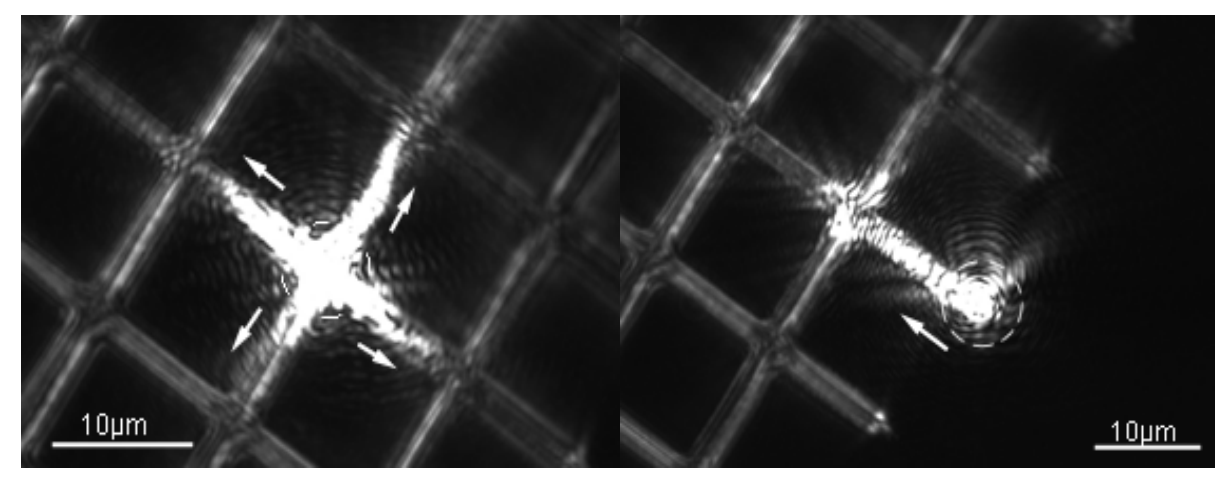

Figure 11. SPPs excited on the crossed part of the crossed-waveguide structure (left), SPPs excited on one port of the crossed-waveguide structure (right). The left image shows SPPs transmitted along the four sides of the structure while the right shows SPPs spreading to the other three sides after excitation on one port

We also fabricated circular structures using TPP with a line width of $800 \mathrm{~nm}$ and diameter of $20 \mu \mathrm{m}$, as in Figure 12. Focusing the laser on different sites on the circle excited different SPPs as shown in the left, middle, and right images in Figure 12. These correspond to the laser position inside, on, and outside of the circle. When the laser position is inside the circle, the SPPs are almost in the inner circle with a short transmission distance in anticlockwise direction; with the laser on the circle, the SPPs are almost evenly distributed on both sides with the anticlockwise transmission in the inner and the clockwise transmission in the outer; with the laser outside the circle, the SPPs localize in good condition and almost concentrate in the outer circle with anti-clockwise transmission. With the SPPs excited in the outer circle, we can focus the plasmons by changing the position of the laser beam focus. Placing the laser beam focus below or above the gold/ORMOCER interface will change the intensity of SPPs to adjust the plasmon focus distance. The exciting laser spot indicated by the dashed circles in Figure 12 is about $4 \mu \mathrm{m}$. If the spot decreases, the SPP focusing effect becomes less pronounced, since the interface area excited by the laser beam decreases. The results show that the circular structure can be used to control the distribution and transmission of SPPs as long as the structure of the component and the excitation location of the laser are well designed. 


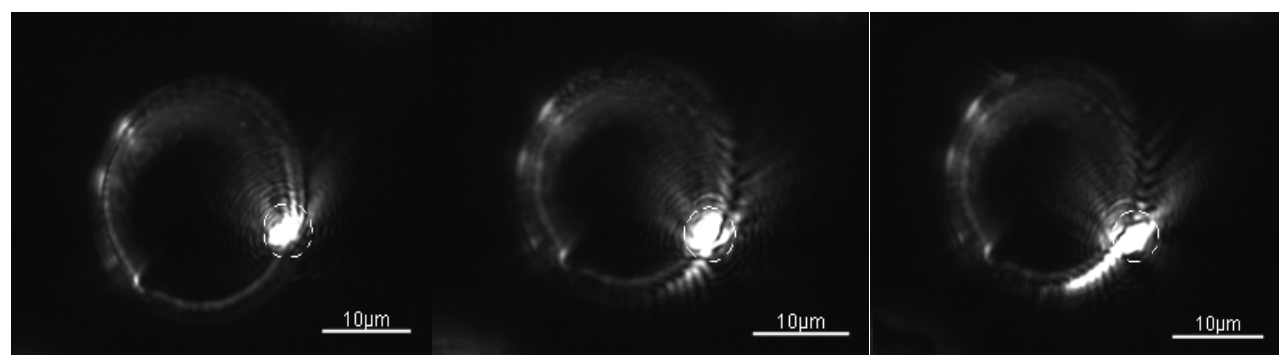

Figure 12. SPPs excited inside the $20 \mu \mathrm{m}$ diameter circular structure (left), SPPs excited on the circular structure (middle), and SPPs excited outside the circular structure (right)

\section{Conclusion}

The combination of the fabrication system and the probe system in the same experimental setup has been studied. With the combination system, different micro and nano ORMOCER structures covered by gold have been fabricated by TPP with the femtosecond laser and the properties of the plasmon components with the structured interfaces have been investigated from excitation to observation. The fabricated plasmonic components show good quality and the excited SPPs show effective excitation and focusing. The results on the ease of fabrication of components, the excitation and manipulation of SPPs and the simplicity of the fabrication system and the probe system provide good references for further studies on plasmon components.

\section{Acknowledgement}

This work was supported by the Jiangsu Natural ScienceFoundation under Grant CX10B_258Z, China Postdoctoral Science Foundation funded project 20100481093, National Natural Science Foundation of China under Grant No. 50975129.

\section{References}

Barnes, W. L., Dereux A., \& Ebbesen T. W. (2003). Surface plasmon sub-wavelength optics. Nature, 424, 824830. https://doi.org/10.1038/nature01937

Bouhelier, A., Huser, Th., Tamaru H., Guentherodt, H. J., Pohl, D. W., Baida, F. I., \& Van- Labeke, D. (2001). Plasmon optics of structured silver films. Physics Review B, 63, 155404. https://doi.org/10.1103/PhysRevB. 63.155404

Chang, S. H., \& Gray, S. K. (2005). Surface plasmon generation and light transmission by isolated nanoholes and arrays of nanoholes in thin metal films. Optics Express, 13, 3150-3165. https://doi.org/10.1364/OPEX.13. 003150

Deubel, M., \& Freymann, G. V. (2004). Direct laser writing of three-dimensional photonic-crystal templates for telecommunications. Nature, 3, 444-447. https://doi.org/10.1038/nmat1155

Drezet, A., Stepanov, A. L., Ditlbacher, H., Hohenau, A., Steinberger, B., Aussenegg, F. R., Leitner, A., \& Krenn, J. R. (2005). Surface plasmon propagation in an elliptical corral. Applied Physics Letters, 86, 209901-10. https://doi.org/10.1063/1.1870107

Evlyukhin, A. B., Bozhevolnyi, S. I., Stepanov, A. L., \& Krenn, J. R. (2006). Splitting of a surface plasmonpolariton beam by chains of nanoparticles. Applied Physics B, 84, 29-34. https://doi.org/10.1007/ s00340-006-2163-8

González, M. U., Weeber, J.-C., Baudrion, A.-L., Dereux, A., Stepanov, A. L., Krenn, J. R., Devaux, E., \& Ebbesen, T. W. (2006). Design, near-field characterization, and modeling of $45^{\circ}$ surface-plasmon Bragg mirrors. Physics Review B, 73, 155 416. https://doi.org/10.1103/PhysRevB.73.155416

Hecht, B., Bielefeldt, H., Novotny, L., Inouye, Y., \& Pohl, D. W. (1996). Local Excitation, Scattering, and Interference of Surface Plasmons. Physics Review Letters, 77, 1889-1892. https://doi.org/10.1103/ PhysRevLett.77.1889

Kaneko, K., Sun, H. B., Duan, X. M., \& Kawata, S. (2003). Two-photon photoreduction of metallic nanoparticle gratings in a polymer matrix. Applied Physics Letters, 83, 1426-1428. https://doi.org/10.1063/1.1601302

Müller, R., Ropers, C., \& Lienau, C. (2004). Femtosecond light pulse propagation through metallic nanohole arrays: The role of the dielectric substrate. Optics Express, 12.5067-5081. https://doi.org/10.1364/OPEX.12.005067 
Qi, M., Lidorikis, E., \& Rakich, P. T. (2004). A three-dimensional optical photonic crystal with designed point defects. Nature, 429, 538-542. https://doi.org/10.1038/nature02575

Raether, H., (1988). Surface Plasmons on Smooth and Rough Surfaces and on Gratings. Springer Tracts in Modern Physics, Springer, Berlin. https://doi.org/10.1007/BFb0048317

Reinhardt, C., Passinger, S., Chichkov, B. N., Marquart, C., Radko, I. P., \& Bozhevolnyi, S. I.(2006). Laserfabricated dielectric optical components for surface plasmonpolaritons. Optics Letters, 31, 1307-1309. https://doi.org/10.1364/OL.31.001307

Stepanov, A. L., Krenn, J. R., Ditlbacher, H., Hohenau, A., Drezet, A., Steinberger, B, Leitner, A., \& Aussenegg, F. (2005). Quantitative analysis of surface plasmon interaction with silver nanoparticles. Optics Letters, 30, 1524-1526. https://doi.org/10.1364/OL.30.001524

Sun, H. B., Matsuo, S., \& Misawa, H. (1999). Three-dimensional photonic crystal structures achieved with twophoton-absorption photopolymerization of resin. Applied Physics Letters, 74, 786-788. https://doi.org/10. $1063 / 1.123367$

Yang, G., \& Zhou, J. (2013).The annealing effect on optical properties of silver copper alloy films. Applied Mechanics and Materials, 320, 329-335. https://doi.org/10.4028/www.scientific.net/AMM.320.329

Zhou, M., Kong, J. J., Yang, H. F., Yan, F., \& Cai, L. (2007). Rapid Fabrication of Three-Dimensional Woodpile Photonic Crystals by Means of Two-Photon Photopolymeization. Chinese Physics Letters, 24, 31643167.https://doi.org/10.1088/0256-307X/24/11/040

\section{Copyrights}

Copyright for this article is retained by the author(s), with first publication rights granted to the journal.

This is an open-access article distributed under the terms and conditions of the Creative Commons Attribution license (http://creativecommons.org/licenses/by/4.0/). 